Published in final edited form as:

Eickelmann, S., Danglad-Flores, J., Chen, G., Miettinen, M. S., \& Riegler, H. (2018). Meniscus shape around nanoparticles embedded in molecularly thin liquid films. Langmuir, 34(38), 1136411373. doi:10.1021/acs.langmuir.8b02266.

\title{
Meniscus shape around nanoparticles embedded in molecularly thin liquid films
}

Stephan Eickelmann, José Danglad-Flores, Guoxiang Chen, Markus S. Miettinen and Hans Riegler

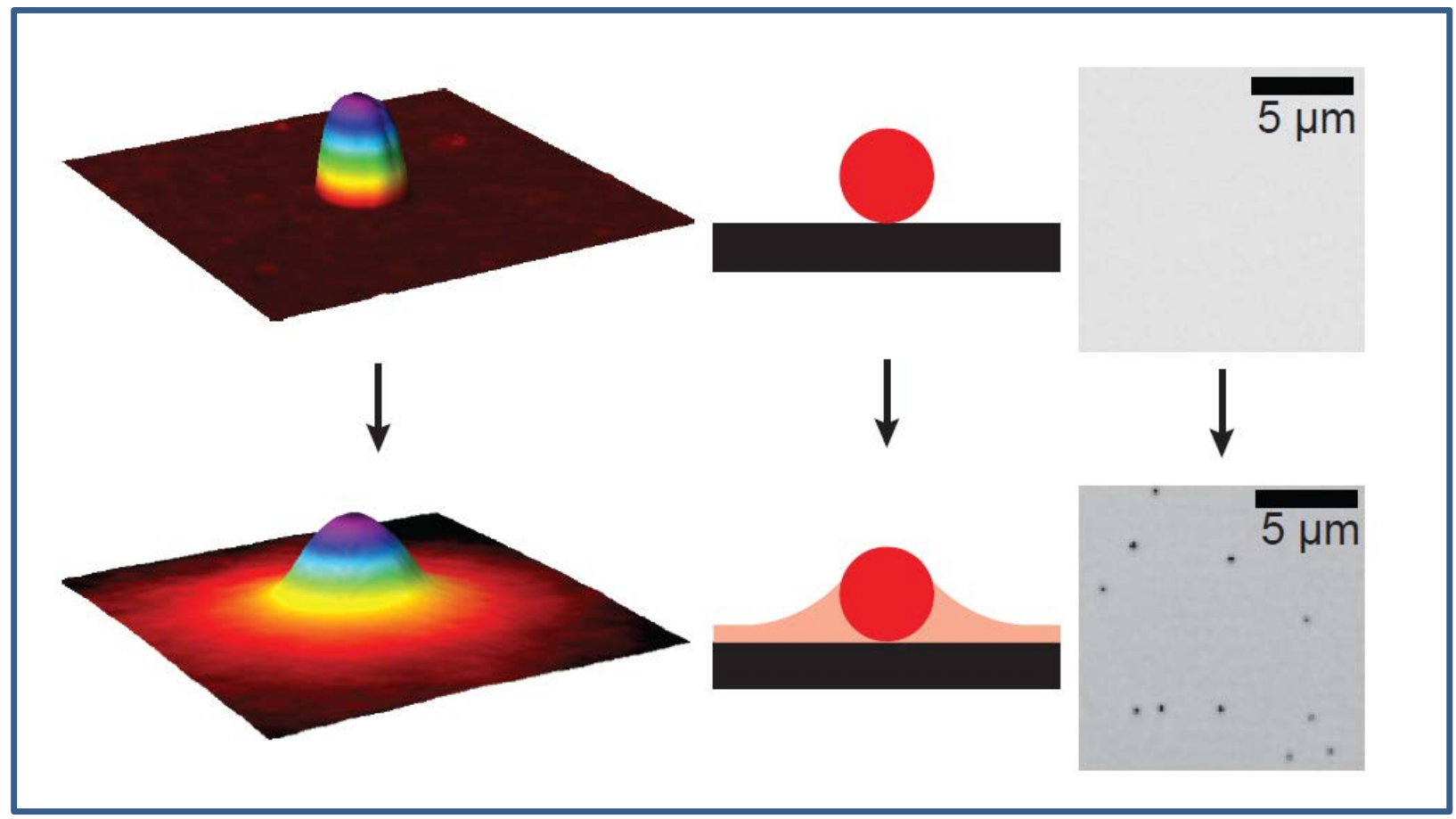




\title{
Meniscus shape around nanoparticles embedded in molecularly thin liquid films
}

\author{
Stephan Eickelmann, ${ }^{*, \dagger}$ José Danglad-Flores,,,$++\Phi$ Guoxiang Chen, ${ }^{\ddagger}$ Markus S. \\ Miettinen, ${ }^{\ddagger}$ and Hans Riegler, ${ }^{*} \ddagger$ \\ $\dagger$ †ax Planck Institute of Colloids and Interfaces, Biomolecular Systems, Science Park \\ Golm, 14424 Potsdam, Germany \\ $\ddagger$ Max Planck Institute of Colloids and Interfaces, Theory and Bio-Systems, Science Park \\ Golm, 14424 Potsdam, Germany \\ ФTechnical University Berlin, Strasse des 17. Juni 135, 10623 Berlin, Germany \\ E-mail: stephan.eickelmann@mpikg.mpg.de; JoseAngel.Danglad-Flores@mpikg.mpg.de; \\ hans.riegler@mpikg.mpg.de
}

\section{Abstract}

Individual nanoparticles embedded in molecularly thin films at planar substrates and the resulting film surface distortion (meniscus) adjacent to the nanoparticles are investigated by conventional optical reflection microscopy. Even for nanoparticles much smaller than the Rayleigh diffraction limit the meniscus creates such a pronounced optical footprint that the location of the nanoparticles can be identified. This is because the decay length (lateral extension) of the meniscus exceeds the size of the nanoparticle by orders of magnitude. Therefore, for the first time, the exact shape of the meniscus of the liquid adjacent to a nano-size object could be measured and analyzed. The meniscus has a zero curvature shape (cosine hyperbolic). The liquid in the meniscus is in pressure equilibrium with the far-field planar film. The decay length decreases with decreasing nanoparticle size. But it is independent from the far-field film thickness. Supposedly the decay length is determined by Van der Waals interactions ( $\mathrm{VdW}$ ) although it is unknown what determines its (unexpectedly large) absolute value. The presented technical approach may be used to investigate biological systems (for instance surface distortions in supported membranes caused by proteins or protein aggregates).

\section{Introduction}

Small objects embedded in thin films are ubiquitous in nature. ${ }^{1-6}$ They are a topic in basic research $^{7-12}$ and proposed as well as used for applications. ${ }^{13-17}$ If the particles are larger than the film thickness, they "stick out". Therefore they will distort the adjacent film surface (cause a "meniscus"). The geometry of this meniscus is of interest in applied and fundamental science, because it reflects the interactions between the particle and its environment and is a measure/cause for the interaction between neighbouring particles. Therefore the meniscus geometry is an important system parameter.

For micrometer-sized and larger systems meniscus shapes have been investigated experimentally and theoretically. ${ }^{18-21}$ For smaller i.e., truly nanometer-size systems, with particle diameters and really thin films (thicknesses $\ll 1 \mu \mathrm{m})$, meniscus geometries have been studied experimentally up to now only for solid systems. For instance, systems with particles embedded in polymeric films were imaged by atomic force microscope (AFM), ${ }^{22-25}$ scanning 
electron microscopy (SEM) ${ }^{22,26}$ or with confocal optical methods. ${ }^{27-33}$ In these cases the meniscus geometries were affected by the solidification process. They may not reflect the equilibrium situation of the particles embedded in the mobile/liquid film. This case, meniscus shapes in the vicinity of nano-size particles embedded in very thin liquid (mobile) films, has not been investigated up to now. The shape of the meniscus of a liquid film adjacent to a nanoparticle (NP) is still unknown. It is still unknown how it depends on particle size, on film thickness, on film properties (surface tension), on substrate properties, etc.

If the size of NPs is well below the resolution limit for visible light (Rayleigh limit ${ }^{34}$ ), their imaging is impossible with conventional optical microscopy. It has been shown however, that it is possible to identify the location of nano size objects with conventional optical microscopy through their refraction/diffraction signature e.g., by the "caustics" caused by surface distortions. ${ }^{35}$ The optical diffraction signature is usually weak but it can be enhanced by sophisticated image processing (oversampling, background subtraction, contrast enhancement, etc. $\left.{ }^{36}\right)$. Slight defocussing along the optical axis $^{37-40}$ may also enhance the diffraction signal in particular with particles embedded in thin films. ${ }^{35,41}$

In the following we present a new approach to (1) determine the location of NPs embedded in a molecularly thin liquid film and (2) measure quantitatively the adjacent meniscus shape. A special interference enhanced optical reflection microscopy setup enables the determination of the location of even very small NPs (diameters $\ll 100 \mathrm{~nm}$ ) embedded in liquid films. The meniscus shape as well as the far-field liquid film thickness are measured with $\mathrm{nm}$ precision. It is found that in comparison to the vertical scale the lateral scale of distortion reaches out further by orders of magnitude more.

\section{MATERIALS AND METHODS}

Solvents and polymers.- As solvents were used: Toluene (99.97 \%, Sigma Aldrich), n-nonane (99.8\%, Sigma Aldrich), ethanol (99,96\%,

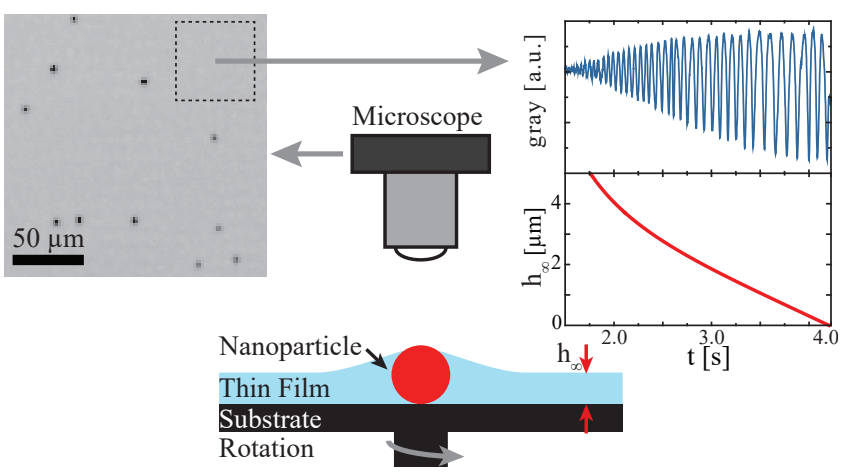

Figure 1: Reflection optical interference microscopy imaging

VWR Chemistry and Chemicals), acetone (99.3 $\%$, J.T. Baker), and water (milliQ). The polystyrene was from Polymer Source Inc (Mw $=45 \mathrm{~kg} / \mathrm{mol}$ ).

Nanoparticles. - Pt-NPs of different diameters $d_{N P}$ were synthesized according to Bigall et $a l^{42}$ (typical size variation of $\pm 20 \%$ (SEM) within batches of the same NP size sorted by centrifugation, see also supplement). They were suspended in water.

Substrates. - Single side polished silicon wafers ((Si(100), p, d=20 cm, Siegert Wafer, Aachen, Germany) with an artificially grown oxide layer of $500 \AA$ (wet oxidation) were cut/broken into substrates of $\approx 2 \mathrm{~cm} \times 2 \mathrm{~cm}$. These substrates were then supersonicated while sequentially submerged each for $5 \mathrm{~min}$ in water, ethanol, acetone, ethanol, and water. Then the substrates were submerged in piranha $\left(\mathrm{H}_{2} \mathrm{SO}_{4}(98 \%) / \mathrm{H}_{2} \mathrm{O}_{2}(30 \%): 3 / 1\right)$ for $30 \mathrm{~min}$ at $70{ }^{\circ} \mathrm{C}$. After copiously flushing with water and sonication in water $(10 \mathrm{~min})$ the samples were finally stored in water for at most $6 \mathrm{~h}$ until use. Directly before use they were dry-blown with nitrogen (purity 5.0).

Film formation by spin casting.- Liquid films were formed on the planar silicon substrates by evaporative spin casting, a well-characterized method to form thin planar films from volatile liquids. ${ }^{43}$ With this method also permanent planar films or permanent coverages of NPs can be prepared in a well-controlled way by deposition of liquid mixtures of volatile and nonvolatile components. ${ }^{44}$

Reflection optical microscopy setup.- Figure 1 shows the experimental setup to optically de- 
termine on-line and locally (within lateral optical resolution) the (time-dependent) thickness of the liquid film during the spin cast process. It allows to determine the local, time-dependent liquid film thickness and the location of individual NPs.

The sample is imaged from the top in reflection mode ${ }^{45}$ (microscope head: Axio Scope.A1 Vario, ZEISS; camera: EoSense@CL MC1362, Mikrotron GmbH; illumination: $6 \mathrm{~W}$, $\lambda=445 \mathrm{~nm}$, LDM-445-6000, LASERTACK, de-speckled by rotational diffusor). With the applied optics (objective (Olympus SLMPLN50x, NA $=0.35$ ) nominally a sample area of $\approx 0.35 \mu \mathrm{m} \times 0.35 \mu \mathrm{m}$ is imaged on a single camera pixel. The setup enables the quasi-stationary, momentary imaging of the (thinning) film at quasi-constant film thicknesses, because typical evaporative film thinning speeds $(1000 \mathrm{~nm} / \mathrm{sec})$ are slow compared to the shortest snap-shot imaging time (1 ms).

Determination of the liquid film thickness.For film thicknesses investigated here, the substrate/film and the film/air interfaces are imaged simultaneously on the camera sensor (their distance is smaller than the depth of focus). Both interfaces reflect some of the illuminating light. Therefore the locally measured (imaged) intensity is modulated by interference effects i.e., the locally imaged intensity depends on the local film thickness (see supplement). With a continuously changing film thickness, the locally imaged intensity is continuously changing. In addition, because the thickness changes during evaporative film thinning are larger than $\lambda /(2 n)(\mathrm{n}=$ refractive index of the film), the recorded reflected intensities oscillate between minima and maxima. As a result, from the relative and absolute local intensity variations in combination with the known refractive indices, a sophisticated imaging software (background subtraction, etc), and an additional interference contrast enhancement through an artificial silica layer on top of the silicon wafer, the local film thickness can be determined with sub-nm resolution ${ }^{45,46}$ (for a discussion of spatial resolution-limiting diffraction effects, see below).

In sum, it should be noted that from the spatio-temporal intensity evolution observed by the camera it is possible to derive the absolute thickness and the absolute time evolution of the undistorted, planar (= laterally uniform intensity), "far-field" film sections $\left(h_{\infty}\right)$ as well as the geometry of the distorted film sections $\left(z(r)=h(r)-h_{\infty}=\right.$ meniscus profile near NPs $)$, at the same time.

Identification of the location of individual NPs. - Individual spherical NPs embedded in a liquid film thinner than the diameter of the NP usually distort the surface of the film adjacent to the NP. The liquid forms a radially symmetrical meniscus, which modifies the locally reflected intensity. The center of this modification identifies the location of the NP.

AFM and thickness of deposited films.- The surfaces of the samples were also imaged and analyzed by AFM (tapping mode, JPK Nanowizard 3, tips from MikroMasch, tip radius $10 \mathrm{~nm}$ (HQ:NSC15/AlBS)). The thickness of dry polymer films was estimated from the depth of scratches performed with the AFM tip. With more precision the thickness of dry polymer films was determined by ellipsometry (M033K001, Physical Instrument, laser: $632.8 \mathrm{~nm}$, sampling area: $0.25 \mathrm{~mm}^{2}$, refraction index of $\mathrm{PS}=1.5842$ ). The index of refraction of the uncoated silicon substrate was measured as 3.855 , for the silica oxide it was 1.457 . The thicknesses of measurements at different locations typically varied by less than $2 \%$.

Preparation of specific NP coverages.- The NPs were deposited by spin casting of aqueous NP suspensions. To achieve a specific NP coverage, $\Gamma_{N P}$, it has been shown ${ }^{43-45}$ that $\Gamma_{N P}=c_{N P} \cdot h_{t r}$, with $\Gamma_{N P}=$ number of NPs per substrate area, $c_{N P}=$ number of NPs per suspension volume. $h_{t r}$ is the so-called "transition height", which marks the system-specific film thickness, below which film thinning is dominated by evaporation. Therefore, all the NPs contained in a film of thickness $h_{t r}$ are finally deposited. This means for instance that a NP coverage of $\Gamma=2.4 \times 10^{9}$ particles $/ \mathrm{m}^{2}$ (equivalent to an average distance between NPs of $20 \mu \mathrm{m}$ ) can be achieved by spin casting an aqueous suspension with a NP concentration of $c_{N P} \approx 5 \times 10^{11}$ particles $/ \mathrm{L}$, because for $h_{t r} \approx$ 
$4.4 \mu \mathrm{m}$ for $\mathrm{H}_{2} \mathrm{O}$ and $\omega=1000 \mathrm{rpm}$.

OPTICAL FOOTPRINTS OF NPS AND THEIR MENISCI IN LIQUID FILMS OF VARIOUS THICKNESSES

Figure 2(a) presents a sequence of interference microscopy snapshots, which were recorded during the evaporative thinning (spin casting) of a toluene film. Before the toluene was cast onto the sample surface already a small amount of individual NPs of different sizes had been attached to the dry silica surface by a preceding, separate spin cast process. The NP coverage was obtained by spin casting an aqueous NP suspension, which contained a mixture of NPs with diameters $d_{N P}$ of $\approx 25,50,100$ and $200 \mathrm{~nm}$. The process conditions and NP-concentrations were adjusted such that the average distance between individual NPs was in the range of several tens of $\mu m$ (see Materials and Methods section).

Figure 2(b) shows how the momentary "farfield" toluene film thickness, $h_{\infty}$ (see Materials and Methods section) evolves with time. $h_{\infty}$ is determined from the time-dependent variation of the "Brightness" (gray level) of an area section with a laterally uniform brightness through the entire evaporation process (i.e., an area where the film thickness is disturbed by a NP).

The data presented in Figure 2(a) show the evaporative thinning of the film of pure toluene, which was spin cast onto a substrate with predeposited NPs. The snapshots reveal that at certain locations spots of locally different gray levels appear as soon as the film thickness has decreased below certain values. The spots remain as the film gets thinner while their gray levels change with changing film thickness. The lateral arrangement of the center of the spots does not change during the toluene film thinning. The spots disappear when the toluene has evaporated completely (spots from the largest NPs with diameters of $200 \mathrm{~nm}$ are still faintly visible even with completely dry substrates). AFM and optical investigations on the lateral arrangements and the surface coverage of the (a)
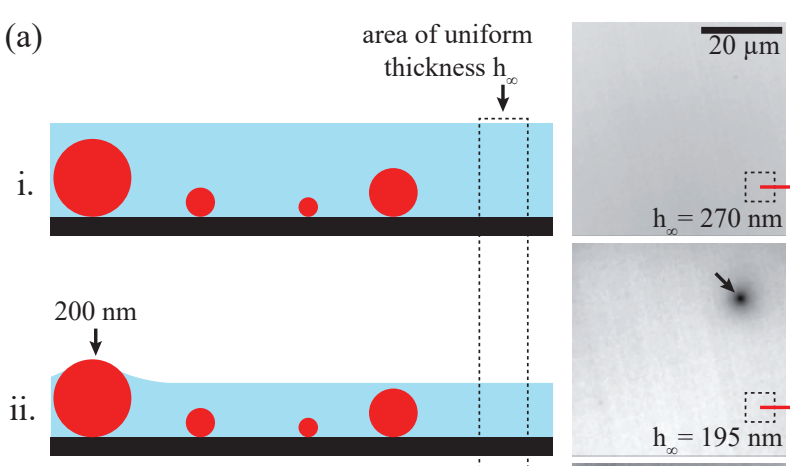

$\mathrm{h}_{\infty}=270 \mathrm{~nm}$
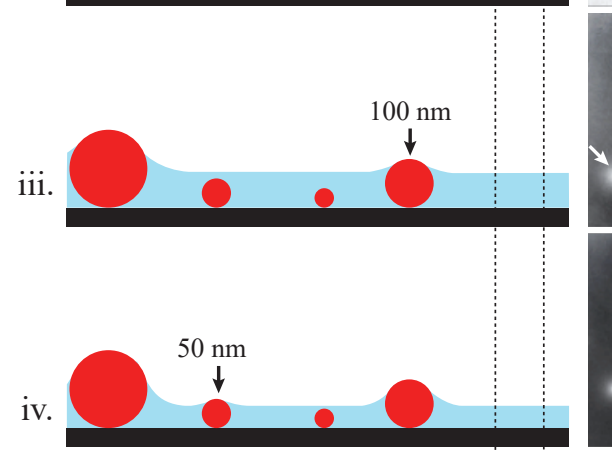

$\mathrm{h}_{\infty}=195 \mathrm{~nm}$
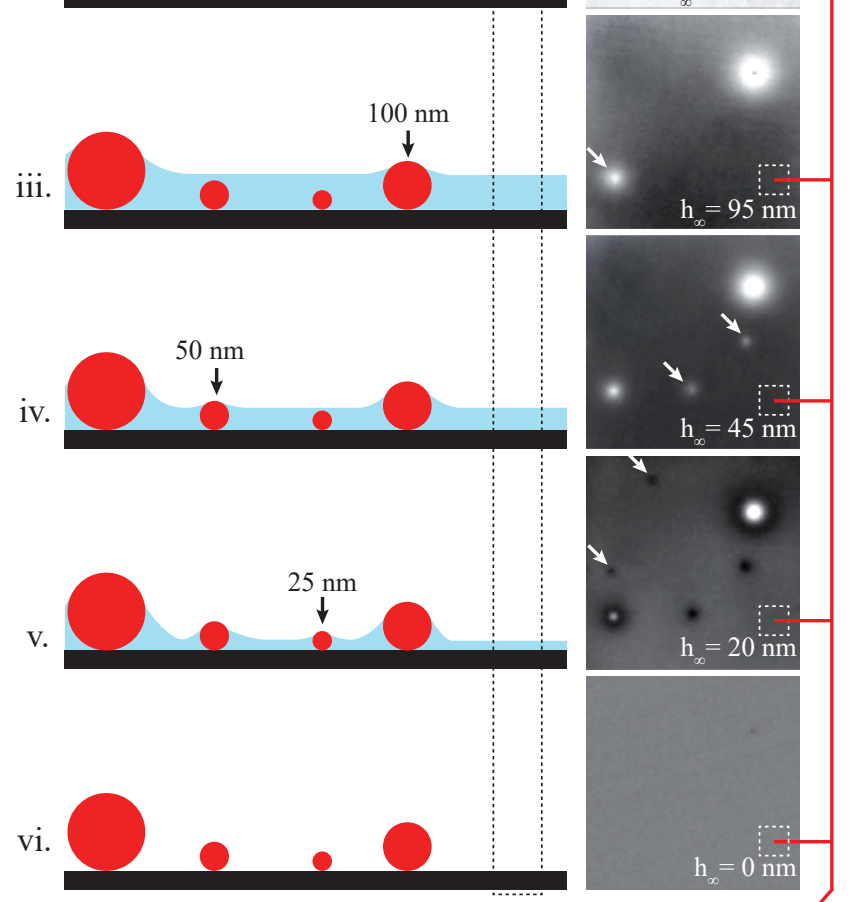

(b)

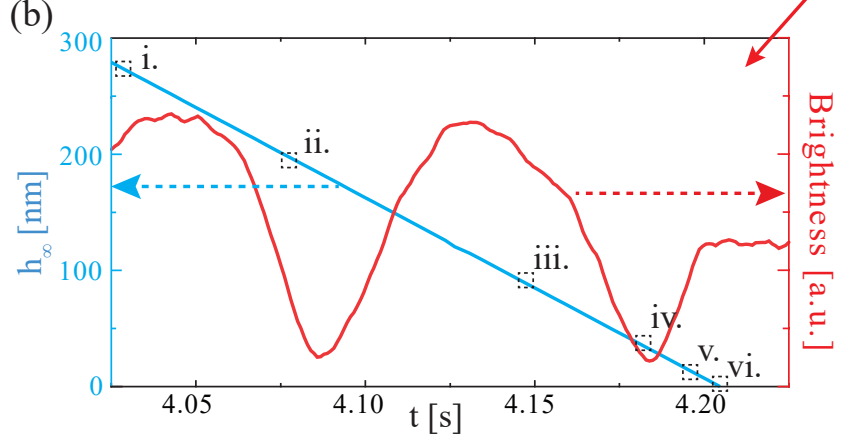

Figure 2: Snapshots of optical footprints of NPs embedded in a thinning toluene film. Attached to the substrate surface are individual NPs with diameters of $d_{N P} \approx 25,50,100$ and $200 \mathrm{~nm}$. The NPs are randomly distributed and laterally several tens of $\mu \mathrm{ms}$ apart. (a) Images recorded at various far-field toluene film thicknesses, $h_{\infty}$, and cartoons of the corresponding vertical film cross sections (not to scale laterally). (b) In red the brightness variation of far-field (planar) film sections with time (due to evaporation) and in blue their corresponding film thickness, $h_{\infty}$. 
NPs on the dry samples prove that individual NPs cause the spots and are located at their center. This is shown by the cartoons next to each microscopy image, suggesting the vertical cross-sections through the liquid films including the NPs. It should be noted that in the cartoons of Figure 2(a) the lateral and vertical scales are different by about a factor of 100 to enable a reasonable visualization of the NPs and their environment.

\section{OCCURRENCE OF OPTICAL FOOTPRINTS AS A FUNC- TION OF THE FAR-FIELD FILM THICKNESS FOR DIF- FERENT LIQUIDS}

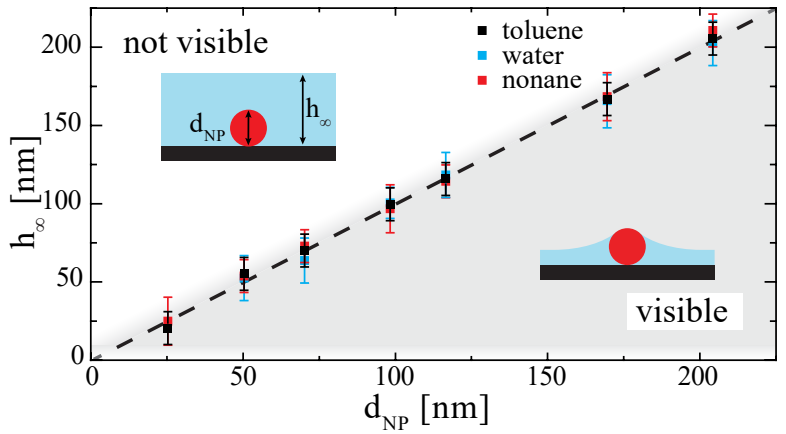

Figure 3: Region where NP footprints are not observed ("not visible") and where footprints are detected ("visible") as function of the NP size $d_{N P}$ and of the far-field film thickness $h_{\infty}$ for various liquids (toluene, water and n-nonane). The dashed line marks $h_{\infty}=d_{N P}$. The Pt-NPs had diameters of 25, 50, 70, 100, 120, 170 and $200 \mathrm{~nm}$.

Figure 3 presents results from optical imaging of substrates during the evaporative thinning of films of various liquids (toluene, water, and n-nonane). The liquids were cast onto the substrates with individual NPs of different diameters $d_{N P}$ attached to the substrate surfaces prior to the liquid deposition. The data are based on measurements analogous to those presented in Figure 2. The Figure shows the range where local optical footprints caused by NPs are observed as a function of the NP size, $d_{N P}$, and of the far-field film thickness, $h_{\infty}$. It reveals that optical footprints of the NPs are only visible if there is a liquid film with $0<h_{\infty} \leq d_{N P}$. For dry samples and for $h_{\infty}>d_{N P}$ optical footprints (spots) such as those presented in Figure 2 are not observed.

\section{MENISCUS PROFILES DE- RIVED FROM THE LOCAL GREY LEVELS}
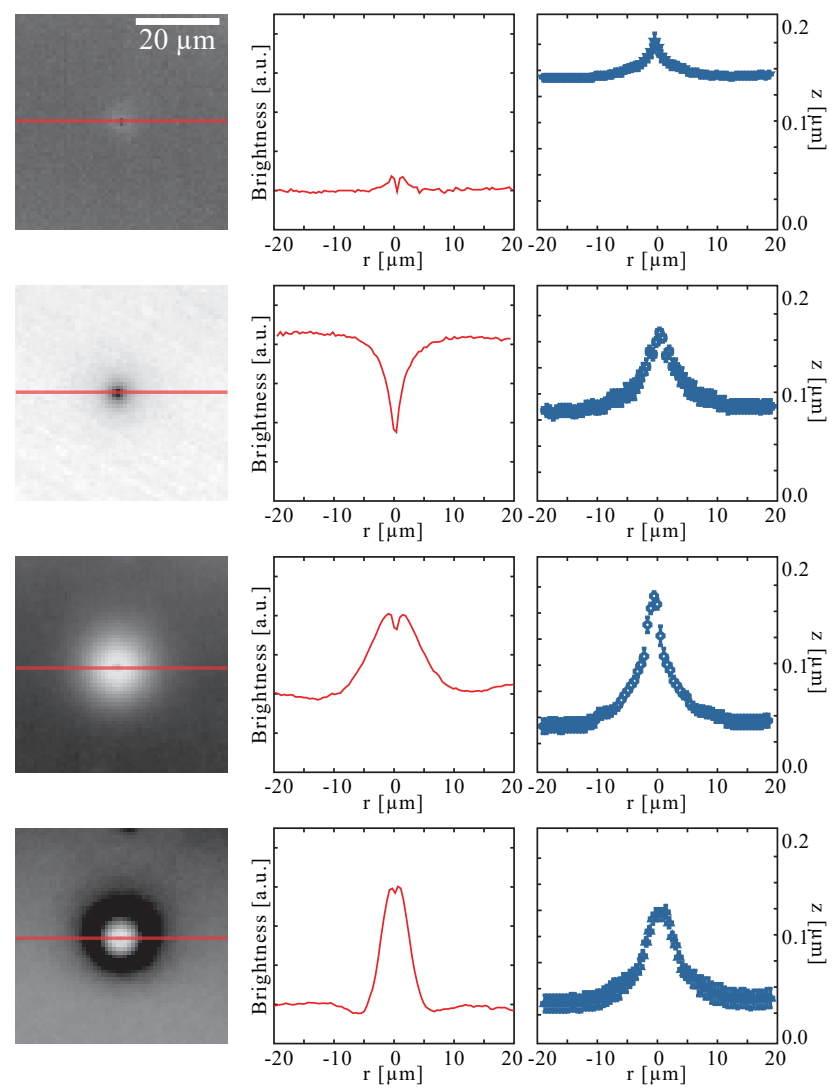

Figure 4: Translation of optical footprint data into meniscus geometries. Depicted is the case of an individual NP $\left(d_{N P}=200 \mathrm{~nm}\right)$ embedded in toluene films of different thicknesses. Left column: Optical reflection images at film thicknesses $h_{\infty}=150,90,50$, and $20 \mathrm{~nm}$. Middle column: Gray level variations along lines through the center of the NP. Right column: Translation of the gray levels of individual camera pixels into the local film thickness. Assumed is the same relation between gray level and film thickness as for planar film sections.

Figure 4 presents the translation of the optical footprint (locally varying gray levels) caused 
by a NP into the geometry of the surrounding meniscus. The NP with a diameter of $200 \mathrm{~nm}$ is embedded in toluene films with far-field thicknesses of $h_{\infty}=150,90,50$, and $20 \mathrm{~nm}$. The column on the left shows the optical images. The column in the middle presents the gray level variations on a line through the center of the location of the NP. The right column depicts the meniscus geometry as it has been derived from the gray level variations. For the derivation of the meniscus profiles it is assumed that the imaged gray level can directly be translated into the local film thickness of the imaged spot (see Figure 2(b) and Methods section). Even though the diameter of the NP is much less than $1 \mu \mathrm{m}$, the meniscus profiles extend up to $\approx 10 \mu \mathrm{m}$ away from the location of the NP (the lateral and the vertical scales of the plots of Figure 4 are different by about two orders of magnitude). The plots show that the optically measured height in the center at $x=0$ does not match the (known) height of the NP. It is different for different $h_{\infty}$, decreasing from about $180 \mathrm{~nm}$ for $h_{\infty}=150 \mathrm{~nm}$ to about $120 \mathrm{~nm}$ for $h_{\infty}=20 \mathrm{~nm}$. This is an optical artifact. The lateral extension of the NP is smaller than the diffraction limit. The data indicate that a oneto-one translation of the gray levels of individual pixels into a corresponding local film thickness is a crude approximation, which may be quantitatively wrong. Therefore the validity of such an analysis has to be investigated. This is done in the following section by a comparison between optical and AFM data.

\section{NPS EMBEDDED IN SOLID POLYMER FILMS: MENISCUS PROFILES DERIVED FROM AFM AND OPTICAL MI-
CROSCOPY}

Figure 5 presents a comparison between AFM and optical microscopy data of the same sample location with NPs embedded in solid polymer films. Individual NPs were first deposited on a planar silica/silicon substrate. Then thin polystyrene (PS) films were spin cast onto these samples from a polystyrene/toluene solution. (a)

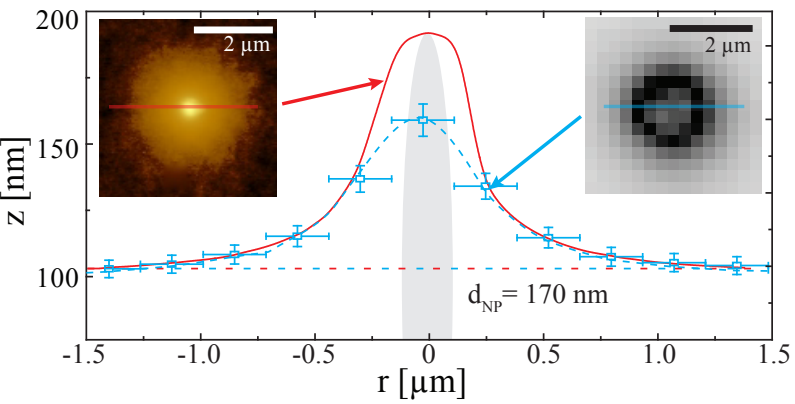

(b)

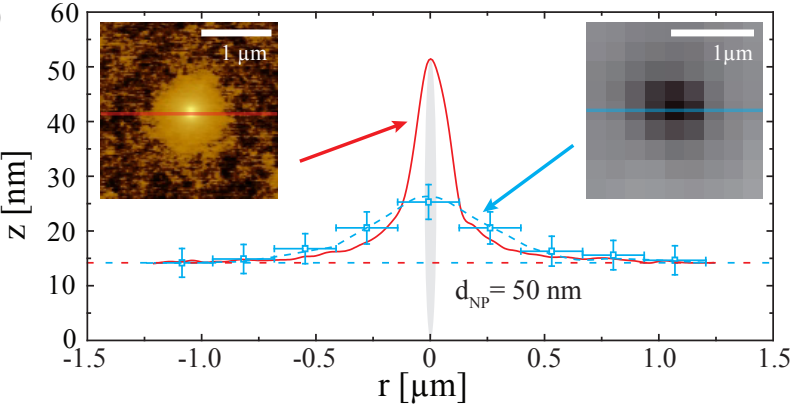

(c)

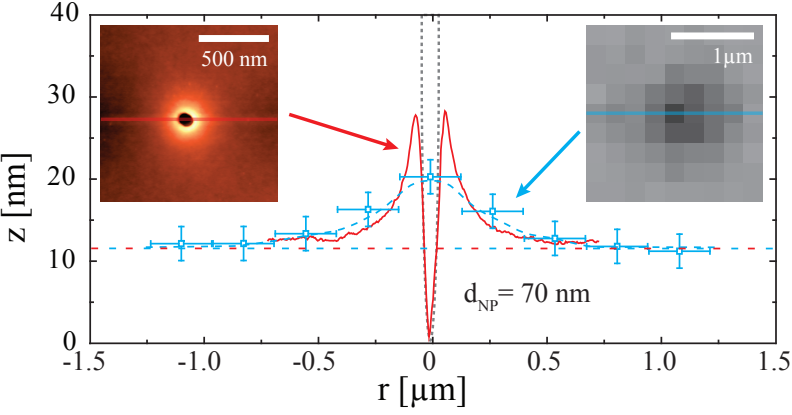

Figure 5: Comparison between the optical and the AFM signatures of NPs embedded in PS films with a) a NP of $170 \mathrm{~nm}$ in diameter $\left(h_{\infty}=100 \mathrm{~nm}\right)$ and b) of $50 \mathrm{~nm}$ diameter $\left(h_{\infty}=\right.$ $15 \mathrm{~nm}) . c)$ the $70 \mathrm{~nm}$ NPs embedded in a PS film $\left(h_{\infty}=12 \mathrm{~nm}\right)$ were removed before AFM investigation. The gray ellipsoid represents the spherical NPs. The optical signatures (blue data points) were derived from the gray levels in a zero-order approach (see main text). The blue dashed line is a guide to the eye. The AFM signature (in red) was obtained by tapping mode. 
The optical and the AFM investigations were performed after complete evaporation of the solvent. Cases (a) and (b) show NPs that are embedded in the PS film. In case (c) the NP embedded in a PS film was removed before the investigation. The figure shows the AFM data (false colour images and height profiles) and optical imaging data (gray level images and interferometrically derived height profiles (see Figures 3 and 4)). The lateral scale is so small that individual camera pixels of uniform gray levels can be identified in the optical images. Nevertheless, the lateral scaling is still much larger $(\mu \mathrm{m})$ than the vertical one $(10 \mathrm{~nm})$, so that the spherical NPs (or their outline as in (c)) indicated in the figure by the gray area appear strongly elliptically distorted.

The data show that by and large the meniscus profiles measured by AFM and by optical interferometry match. Significant differences between the "true" (expected) and the measured profiles appear only within $200 \mathrm{~nm}$ from the NP (or from the center of its former location, as in case (c)). Most likely these differences around $x=0$ are due to diffraction effects in the case of the optical imaging. Very close to the NPs the AFM data may also not reflect the true shape of the surface topography due to the conical shape of the AFM tip (tip convolution effect). In the case of Figure 5(c) a NP of $70 \mathrm{~nm}$ diameter was removed after the polymer film formation. This can clearly be seen from the AFM data, whereas the optical data do not reveal the crater-like shape of the surface.

The data depicted in Figure 5 reveal two important features:

1.) For comparable NP sizes and far-field film thicknesses the lateral range of the meniscus distortion is significantly smaller for the solid polymer films than for liquid films of pure solvents (see Figure 4). This indicates that polymer enrichment in the course of the solvent evaporation during film formation affects the final meniscus shape.

2.) The geometrical meniscus profiles measured by AFM and by optical reflection microscopy are virtually identical within the measurement errors (except for the center region very close to the NP). This means meniscus geometries derived from optical reflection microscopy images are quantitatively correct except for a region of about $0.5 \mu \mathrm{m}$ around the location of the NP.

\section{MENISCUS GEOMETRIES}

Figure 6(a) presents experimental data of the geometry of the meniscus profiles in the vicinity of a NP with a diameter of $d_{N P}=120 \mathrm{~nm}$. For a better visualization of the meniscus distortion the linear scaling of the horizontal and the vertical axes differ by a factor of 80 (the spherical NPs are plotted in scale and therefore appear distorted). The NP was embedded in toluene films with three different far-field liquid film heights, $h_{\infty}=29,42$, and $71 \mathrm{~nm}$ (indicated by the dashed blue lines). The two data sets depicted represent the profiles from either sides of the NP. According to the data the meniscus reaches out as far as $\approx 5 \mu \mathrm{m}$ away on both sides from the NP. Figure 6(b) shows the same data in a lin-log plot. The linear behaviour reveals a logarithmic meniscus profile within most of the meniscus range.

Figure 6(c) shows a schematic of the cross section through the NP and the film/meniscus in the vicinity of the NP. It presents the definitions of the various parameters that are used to fit the experimental data. It turns out that all meniscus shapes can be fitted quite well by a combination of a logarithmic (hyperbolic cosine $)^{47}$ and an exponential decay:

$$
\begin{array}{r}
z(r)=\left[h_{0}-h_{\infty}-r_{0} \cdot \ln \left(\frac{r}{r_{0}}+\sqrt{\left(\frac{r}{r_{0}}\right)^{2}-1}\right)\right] \\
\cdot e^{-\frac{r-r_{0}}{L}}+h_{\infty} .
\end{array}
$$

According to this equation the logarithmic contribution dominates for $r<L$ i.e., in the meniscus region up to where the meniscus phases out into the planar film. In this transient region away from the NP, close to $r=L$, the exponential contribution provides a smooth transition between the essentially logarithmic meniscus shape and the flat film. It should be noted that the profile of Equation 1 formally describes 

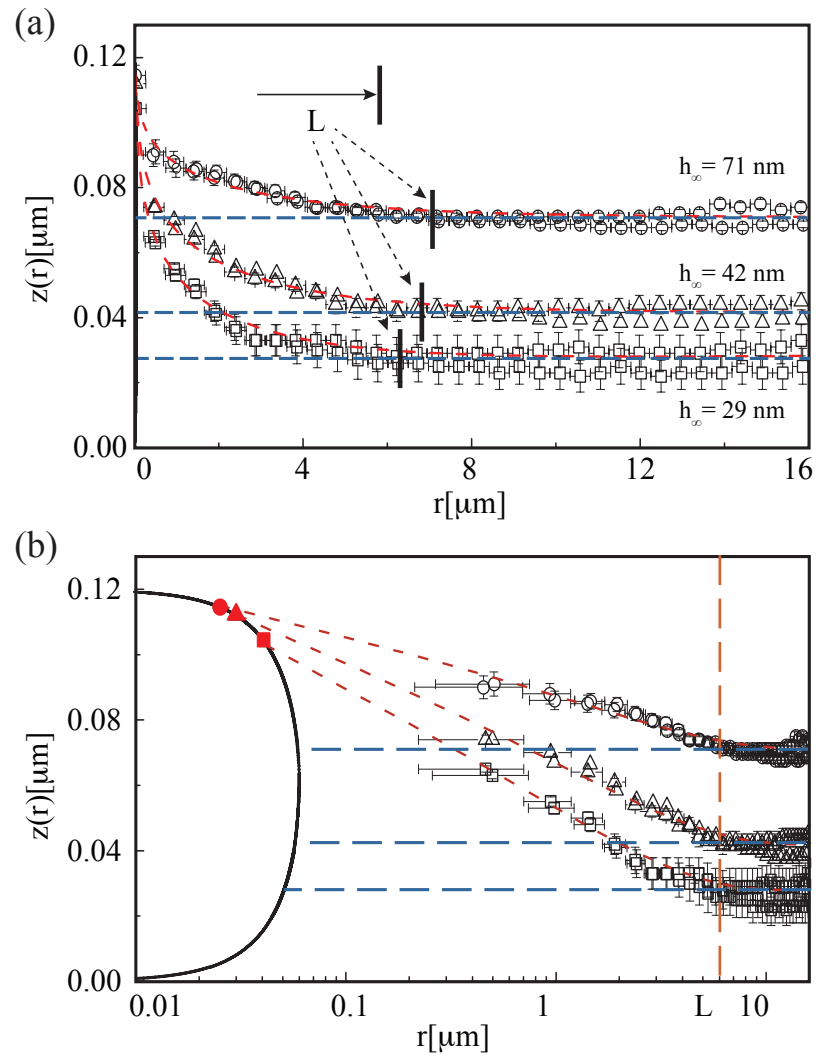

(c)

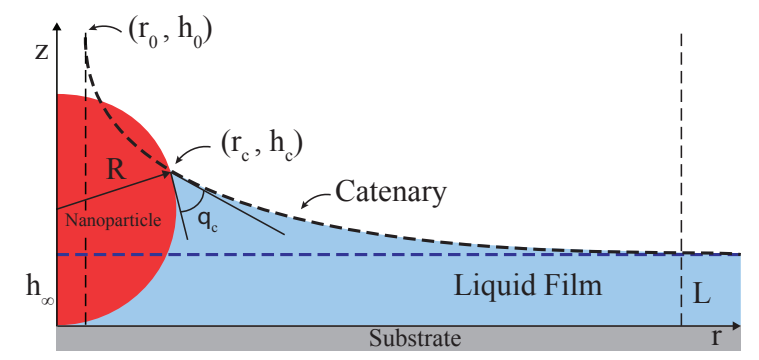

Figure 6: Meniscus geometries derived from optical reflection microscopy. (a) Lin-lin-plot of the meniscus profiles around a NP with $d_{N P}=120 \mathrm{~nm}$, embedded in toluene films of $h_{\infty}=29 \mathrm{~nm}, 42 \mathrm{~nm}$, and $71 \mathrm{~nm}$ as indicated by the blue dashed lines. Each curve consists of two sets of data points (from both sides of the NP) The red dashed lines show a hyperbolic cosine fit of the data points. The vertical and horizontal scales are different by about a factor of 80. (b) Lin-log-plot of the data shown in (a). (c) Cartoon of the assumed meniscus profiles and definition of the various parameters. a meniscus shape with its left vertex contacting an (imaginary) vertical round cylinder of radius $r=r_{0}$ at a height $z=h_{0}$ with a contact angle of $\Theta_{c}^{c y l}=0$. Mathematically this profile is identical to a meniscus, which intersects a NP with radius $R$ at $r=r_{c}$ and at a height $z=h_{c}$ with a contact angle $\Theta_{c}^{N P}$ between the liquid and the NP surface (for details see supplemental material).

Figures 6(a) and (b) demonstrate that the experimental data can nicely be fitted (red dashed curves) with the profile described by Equation 1. The linear-logarithmic plot Figure 6(b) shows a linear behaviour in virtually the entire meniscus range between the contact with the $\mathrm{NP}$ and the transition to the planar far field film surface. Practically, for fitting the experimental data, the exponential contribution of Equation 1 is rather negligible. It is only necessary to formally provide a smooth connection between the logarithmic meniscus profile and the planar far-field film surface. Figure 6 (b) also shows that fits of the experimental data with meniscus profiles according to Equation 1 yield rather well-defined distortion lengths $L$. Regarding interfacial physics, the logarithmic meniscus shape means that the liquid surface on the meniscus has nowhere a net local curvature. Hence there is no capillary pressure difference between the liquid in the meniscus section and the liquid within the surrounding planar film.

\section{UNIVERSAL MENISCUS PRO- FILE AND DISTORTION LENGTH \\ $\mathbf{L}$}

All the individually measured experimental meniscus profiles can be fitted quite well with the profile described by Equation 1, yielding different $L, \Theta_{c}, h_{c}, r_{c}$, as well as $h_{0}$ and $r_{0}$ for the different known/measurable $R$ and $h_{\infty}$. Because all menisci can universally described by Equation 1 they also can be plotted in a universal way with proper axis scaling. This is demonstrated by Figure 7 presenting a large number of experimental data from different sized NPs embedded in films of different thicknesses. The films consisted of (a) toluene and (b) water. All 

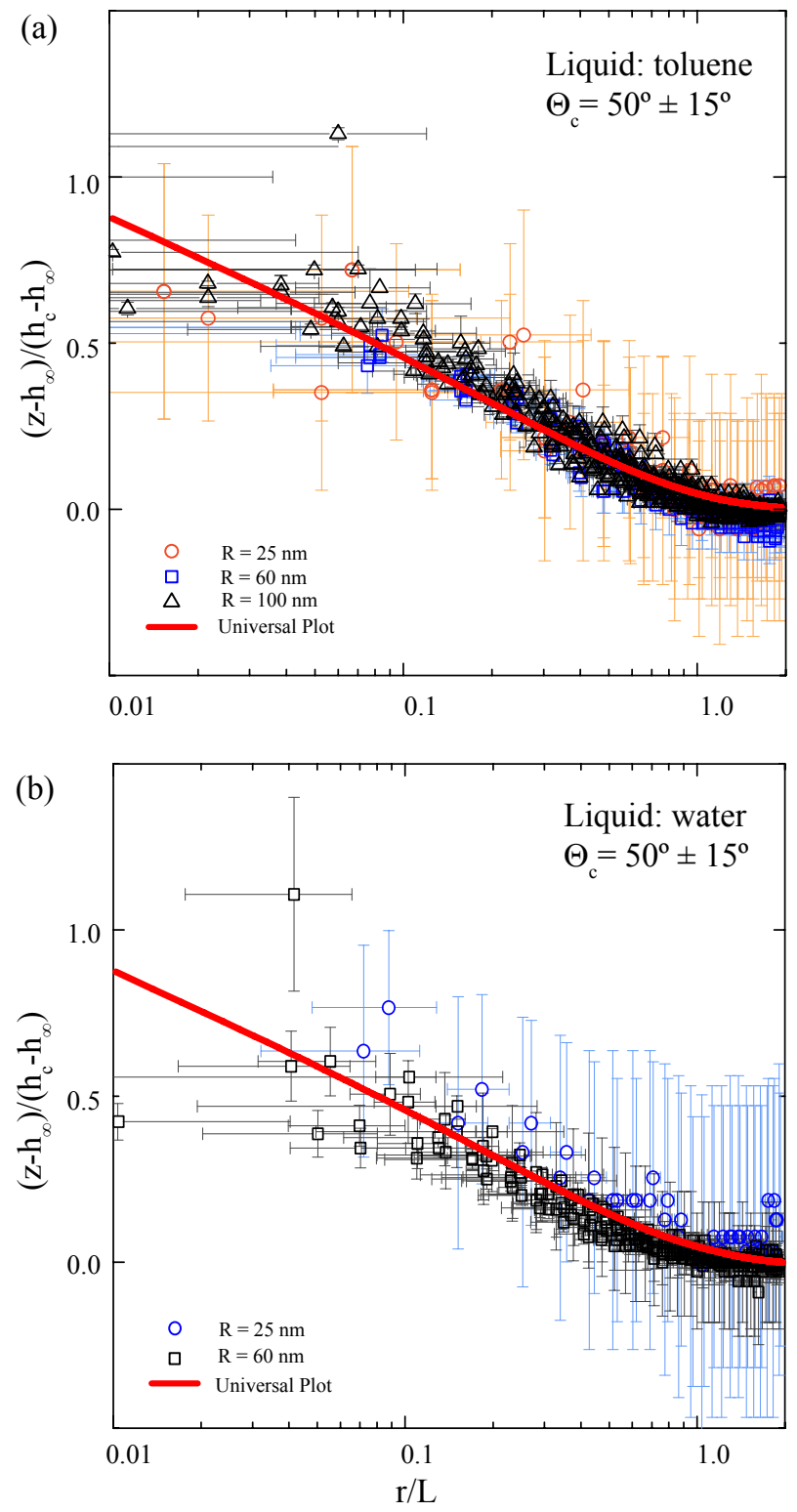

Figure 7: Meniscus geometries as measured for different NP radii, $R$, and film thicknesses, $h_{\infty}$. The data are fitted according to the profile presented in Figure 6 and plotted in a universal way by rescaling the $\mathrm{x}$-axis (lateral distance from the center of the NP, $r$ ) with the distortion length, $L$, and the y-axis (net vertical meniscus elevation, $z-h_{\infty}$ ) with the net distance between the film surface and the contact with the particle at $h_{c}-h_{\infty}$. Singled out by colour are those data points, where the interference conditions lead to a rather weak thickness contrast (orange data points in (a) and blue data points in (b)). Although technically this leads to rather large systematic error bars, the data still reflect the general behaviour quite well. the fits indicate for both liquids the same contact angle with the NPs of $\Theta_{c} \approx 50 \pm 10 \mathrm{deg}$.

Figure 8(a) shows a plot of the distortion lengths $L$ vs the NP radii $R$. $L$ is derived from fits of the experimental measured meniscus profiles. It can be seen that $L$ increases with $R$ in a slightly non-linear fashion (assuming that the origin is a data point). It is also observed that $L$ of water is always slightly larger than $L$ of toluene. Quite remarkable, $L$ is independent from $h_{\infty}$.

Figure 8(b) illustrates the findings presented in Figure 8 through a combination of raw imaging data from real experiments and schematics, which show the corresponding cross sections of the liquid film plus NP in the same lateral scale. Depicted are the cases of $d_{N P}=200 \mathrm{~nm}$ and $h_{\infty}=90 \mathrm{~nm}$ as well as the combination of $d_{N P}=50 \mathrm{~nm}$ and $h_{\infty}=12 \mathrm{~nm}$. The lateral scales of all microscope images and cartoons are the same. The vertical scales in both cartoons are the same but different to the corresponding lateral scales (hence the strongly distorted shape of the spherical NPs).

\section{DISCUSSION AND CON- CLUSION}

We demonstrate that it is relatively easy to locate with conventional optical reflection microscopy small, individual NPs, if they are embedded in films about as thick or thinner than their diameter. Their location can be detected by conventional optical microscopy although they are much smaller than the Rayleigh diffraction limit. This is because the NPs distort the adjacent film surface and it turns out that this surface distortion ("meniscus") reaches out laterally orders of magnitude further than the size/diameter of the NPs. The unexpectedly large meniscus region creates a rather pronounced optical "footprint" and thus enables the tracking of the NP location. The NPs are located at the center of the radially symmetrical surface distortions. They do not move laterally during the evaporative film thinning.

The configuration of individual NPs attached 
(a)

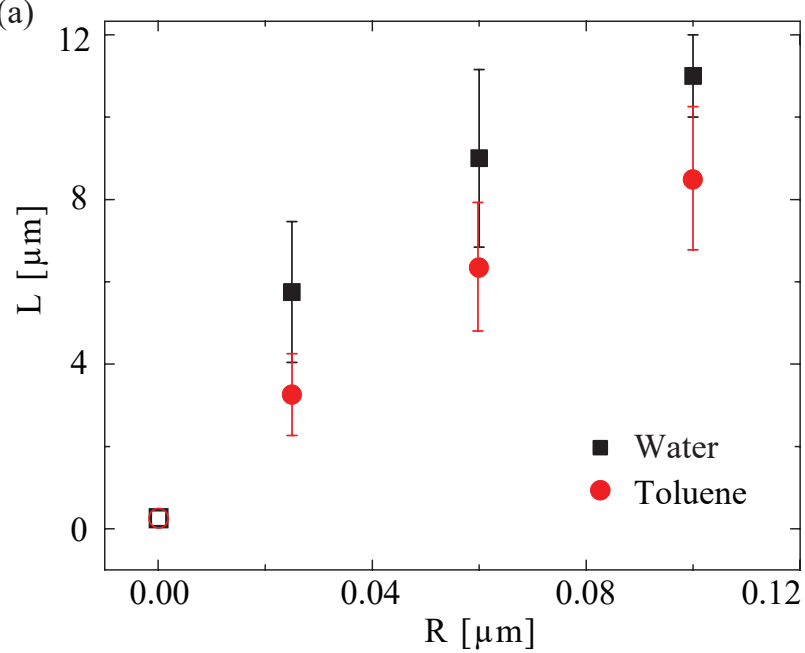

(b)
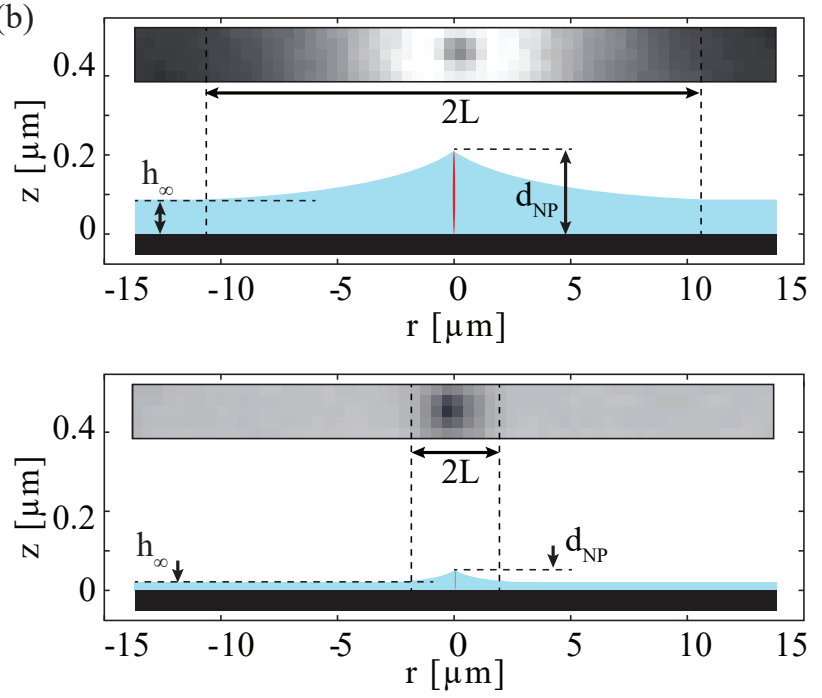

Figure 8: (a) Distortion length $L$ vs NP radius $R$ as derived from the fits of the experimental data. The open circles/squares at the origin indicate the data that are expected at this point. (b) Optical imaging raw data and corresponding cartoons of the derived meniscus shapes for the combination of $d_{N P}=200 \mathrm{~nm}, h_{\infty}=90 \mathrm{~nm}$ (top) and $d_{N P}=50 \mathrm{~nm}, h_{\infty}=12 \mathrm{~nm}$ (bottom). The lateral and the vertical scales are different by nearly two orders of magnitude (the NPs appear as very elongated red ellipses). to a planar surface and embedded in a film of a thickness similar to the diameter of the NP or less is investigated in detail. To this end planar substrates with attached individual NPs are coated by evaporative spin casting with continuously thinning planar liquid films. During film thinning the substrate surface is investigated with reflection optical microscopy. A combination of interferometry and optical imaging provides the local film thickness with nanometer (vertically) and micrometer (laterally) resolution, respectively. Because the lateral extend of menisci caused by even rather small NPs exceeds micrometers, their geometry can be measured optically. This is tested and confirmed by calibration experiments with AFM for the case of solid polymer films.

The meniscus shapes are measured and analyzed as a function of 1.) the diameter of the NPs, 2.) the far-field thickness of the surrounding films, 3.) the composition (surface tension) of the films, and 4.) the state (solid vs liquid) of the films. It turns out that the decay length $L$ of the menisci depends on the size of the NPs, $d_{N P} \cdot{ }^{21} L$ increases non-linearly with the NP size, from a few $\mu m$ for NPs with $25 \mathrm{~nm}$ diameter to about $10 \mu \mathrm{m}$ for NPs with $200 \mathrm{~nm}$ diameter. $L$ is independent from the far field film thickness $h_{\infty}$. In general $L$ is slightly higher for water films compared to toluene films. For solid polymer films $L$ is substantially smaller than for the liquid films. This may be attributed to polymer enrichment processes in the late stages of film drying, because $L$ of the liquid polymer/solvent solutions during solvent evaporation is similar to the one of the pure solvent (toluene). Even in the cases of the system with pure solvents evaporation may influence the meniscus shape ${ }^{48}$ (and vice versa) e.g., through a locally varying evaporative flux because of the meniscus shape ("coffee ring effect" 49 ). However, this appears unlikely, because in real scale the menisci are nearly flat and the net meniscus curvature is zero.

All menisci can be fitted with a cosine hyperbolic profile (except for the region at the transition to the planar film, which can be fitted with a decaying exponential). The cosine hyperbolic profile means that there is no net curvature in 
the meniscus region. Therefore there is no capillary pressure i.e., the liquid in the meniscus region is in pressure equilibrium with the liquid of the far-field planar film.

The most unexpected result is the magnitude of $L$. In macroscopic systems, where gravitation plays a role, the capillary length (typically millimeters) is a measure for $L$. On the other hand, if the size of the particle causing a surface distortion becomes vanishingly small, it can be assumed that $L$ also becomes vanishingly small. For the intermediate range, with nanosize objects distorting the film surface, gravitation can safely be neglected. $L$ has never been measured up to now in this range and it is not clear what governs its size. ${ }^{21}$ It can be assumed that in cases with uncharged, organic liquids (toluene) VdW interactions (disjoining pressure) determine the meniscus shape. Yet the disjoining pressure changes non-linearly with film thickness. Therefore one would expect that the farfield thickness $h_{\infty}$ has a significant influence on $L$. However, this is not the case. In addition, there is also no difference in $L$ for substrates with thin silica layers $(1.5 \mathrm{~nm}$, natural oxide layer) and those with artificially grown thick $(50 \mathrm{~nm})$ silica layers, although this should make a difference in the Hamaker constant. On the other hand, $L$ is significantly different for water and toluene.

The logarithmic meniscus shape and the distortion lengths $L$ are quantitatively quite reliable measure, because they are derived directly from experimental data. This holds in particular for the distortion lengths $L$. It can be guessed already with some confidence $(10-20 \%)$ from the raw data without any extra explicit data analysis (see for instance the width of the "spots" in Figures 2(a) and 4). The numbers for the contact angles, $\Theta_{c}^{N P}$, on the other hand, result from the extrapolation of the meniscus profiles towards the NPs and the contact of this profile with NPs that are assumed perfectly spherical with perfectly smooth surfaces (see supplement). In view of this rather indirect method and its sensitivity to relatively small variations of the meniscus profile, the contact angles are not in the focus of this report. The data indicate that toluene and water may have the same contact angle of $\Theta_{c} \approx 50 \pm 10 \mathrm{deg}$. But this result should not be overrated and needs further investigation.

The shape of the meniscus and its decay length $L$ is scientifically of great interest. It reflects and thus reveals the force (energy) conditions in the vicinity of the NPs. Beyond that, it is important because of the capillary interactions between neighbouring NPs through the interference between neighbouring menisci. For instance, if $L$ is in the range of $\mu \mathrm{m}$ even for NPs as small as only several tens of $\mathrm{nm}$, then neighbouring NPs of this size will interact with each other over a distance orders of magnitude larger than their own size and far beyond the range of direct $\mathrm{VdW}$ interactions.

The investigated model system of individual spherical NPs attached to planar surfaces coated with liquid films of well defined thicknesses might at first sight appear quite irrelevant for "real" systems. However, it refers to configurations occurring very often in nature as well as in applications. Ubiquitous examples are (thinning) liquid films close to the perimeter of drying drops or at the perimeter of retreating (evaporating) flat films. Also, the investigations presented here bear some relevance for biological systems. Molecularly thin liquid films distorted by embedded NPs are similar to (supported) biological membranes that are locally "distorted" by embedded "particles" such as proteins or protein aggregates. Both, the sizes of the film/membrane thicknesses as well as the sizes of the causes of the distortion can be comparable. Thus it is conceivable, that based on the approach presented here, one might directly be able to measure the lateral extension of the membrane thickness distortions caused by protein aggregates.

In conclusion, we show how to image with optical reflection microscopy the location of individual NPs attached to planar substrates embedded in liquid films as thin or thinner than the size of the NPs. The NPs are much smaller than the Rayleigh diffraction limit. Nevertheless, they can be detected optically, because they create a distortion of the adjacent film surface (meniscus), which extends laterally over orders of magnitude further than the size of the 
NPs. Therefore the meniscus shape can even be measured by optical interferometry. The meniscus has cosine hyperbolic (zero net curvature) shape. There is no (capillary) pressure difference between the liquid of the far field planar film and the liquid in the meniscus region. It is unknown what determines the decay length of the meniscus. The decay length is independent from the thickness of the far field planar film. It decreases with decreasing size of the NP. The observation and measurement technique reveals the meniscus shapes of films of nanometer thickness caused by embedded nano size object. Therefore it might be useful for the investigation of biological (supported) membranes with proteins (protein aggregates) as distortion sources.

Acknowledgement Thanks to Reinhard Lipowsky for institutional and financial support. JDF was funded by the DAAD and DFG through the IRTG 1524, and GC was funded by the Chinese Scholarship Council.

Authorship Declaration: SE, JDF, and GC performed the experiments and contributed equally. SE focused on the microscopy imaging. He worked on the image processing and translated the optical raw data into film thicknesses. JDF performed the AFM measurements and did the analysis/fitting of the the meniscus data obtained by AFM and optical microscopy. GC also performed some of the optical microscopy experiments and also synthesized and characterized the nanoparticles. MSM worked on the theoretical background of the meniscus shape. HR organized and supervised the research, wrote the manuscript and contributed to the data analysis and interpretation. All authors have discussed the results, read the manuscript and agreed with its content.

\section{Supporting Information Avail- able}

The following files are available free of charge. S1 - S3: SEM characterization of the homogeneity and size of the Pt-NPs, a detailed description of the analytical expression for the film distortion including its surface shape and the contact the particle, the numerical data analysis, and the calculation of the reflective value according to Fresnel equations.

\section{References}

(1) Tenzer, S.; Docter, D.; Kuharev, J.; Musyanovych, A.; Fetz, V.; Hecht, R.; Schlenk, F.; Fischer, D.; Kiouptsi, K.; Reinhardt, C.; Landfester, K.; Schild, H.; Maskos, M.; Knauer, S. K.; Stauber, R. H. Rapid formation of plasma protein corona critically affects nanoparticle pathophysiology. Nature nanotechnology 2013, 8, 772 .

(2) Meng, F.; Zhang, S.; Oh, Y.; Zhou, Z.; Shin, H.-S.; Chae, S.-R. Fouling in membrane bioreactors: an updated review. Water research 2017, 114, 151-180.

(3) Sheng, Z.; Liu, Y. Effects of silver nanoparticles on wastewater biofilms. $\mathrm{Wa}$ ter Research 2011, 45, 6039-6050.

(4) Bahrami, A. H.; Raatz, M.; AgudoCanalejo, J.; Michel, R.; Curtis, E. M.; Hall, C. K.; Gradzielski, M.; Lipowsky, R.; Weikl, T. R. Wrapping of nanoparticles by membranes. Advances in colloid and interface science 2014, 208, 214-224.

(5) Shang, L.; Nienhaus, K.; Nienhaus, G. U. Engineered nanoparticles interacting with cells: size matters. J Nanobiotechnol 2014, 12, b26.

(6) Jiang, W.; Kim, B. Y.; Rutka, J. T.; Chan, W. C. Nanoparticle-mediated cellular response is size-dependent. Nature nanotechnology 2008, 3, 145.

(7) Weissleder, R.; Nahrendorf, M.; Pittet, M. J. Imaging macrophages with nanoparticles. Nature materials 2014, 13, 125

(8) Choueiri, R. M.; Galati, E.; TherienAubin, H.; Klinkova, A.; Larin, E. M.; Querejeta-Fernndez, A.; Han, L.; 
Xin, H. L.; Gang, O.; Zhulina, E. B.; Rubinstein, M.; Kumacheva, E. Surface patterning of nanoparticles with polymer patches. Nature 2016, 538, 79.

(9) Balazs, A. C.; Emrick, T.; Russell, T. P. Nanoparticle polymer composites: where two small worlds meet. Science 2006, 314, 1107-1110.

(10) Bigioni, T. P.; Lin, X.-M.; Nguyen, T. T.; Corwin, E. I.; Witten, T. A.; Jaeger, H. M. Kinetically driven self assembly of highly ordered nanoparticle monolayers. Nature materials 2006, 5, 265.

(11) Grzelczak, M.; Vermant, J.; Furst, E. M.; Liz-Marzán, L. M. Directed self-assembly of nanoparticles. ACS nano 2010, 4, 3591-3605.

(12) Yunker, P. J.; Still, T.; Lohr, M. A.; Yodh, A. Suppression of the coffee-ring effect by shape-dependent capillary interactions. Nature 2011, 476, 308.

(13) Lizotte, P.; Wen, A.; Sheen, M.; Fields, J.; Rojanasopondist, P.; Steinmetz, N.; Fiering, S. In situ vaccination with cowpea mosaic virus nanoparticles suppresses metastatic cancer. Nature nanotechnology 2016, 11, 295.

(14) Hajipour, M. J.; Fromm, K. M.; Ashkarran, A. A.; de Aberasturi, D. J.; de Larramendi, I. R.; Rojo, T.; Serpooshan, V.; Parak, W. J.; Mahmoudi, M. Antibacterial properties of nanoparticles. Trends in biotechnology 2012, 30, 499-511.

(15) Yeaman, M. R. Platelets in defense against bacterial pathogens. Cellular and molecular life sciences 2010, 67, 525-544.

(16) Peng, J.; Han, Y.; Fu, J.; Yang, Y.; Li, B. Formation of regular hole pattern in polymer films. Macromolecular Chemistry and Physics 2003, 204, 125-130.

(17) Kim, D. J.; Jo, M. J.; Nam, S. Y. A review of polymer-nanocomposite electrolyte membranes for fuel cell applica- tion. Journal of Industrial and Engineering Chemistry 2015, 21, 36-52.

(18) Kralchevsky, P. A.; Paunov, V. N.; B., I. I.; Nagayama, K. Capillary Meniscus Interaction between Colloidal Particles Attachewd to a Liquid-Fluid INterface. Journal of Colloid and lnterface Science 1992, 151, 79.

(19) Kralchevsky, P. A.; Nagayamaa, K. Capillary interactions between particles bound to interfaces, liquid films and biomembranes. Advances in Colloid and Interface Science 2000, 85, 145-192.

(20) Fiegel, J.; Jin, F.; Hanes, J.; Stebe, K. Wetting of a particle in a thin film. Journal of Colloid and Interface Science 2005, 291, 507-514.

(21) Tress, M.; Karpitschka, S.; Papadopoulos, P.; Snoeijer, J. H.; Vollmer, D.; Butt, H.-J. Shape of a sessile drop on a flat surface covered with a liquid film. Soft matter 2017, 13, 3760-3767.

(22) Saito, Y. Q Nanoparticles and Nanocapsules. Carbon Nanotubes: Preparation and Properties 1996, 249.

(23) Zheng, J.; Zhu, Z.; Chen, H.; Liu, Z. Nanopatterned assembling of colloidal gold nanoparticles on silicon. Langmuir 2000, 16, 4409-4412.

(24) Jin, R.; Jureller, J. E.; Scherer, N. F. Precise localization and correlation of single nanoparticle optical responses and morphology. Applied physics letters 2006, 88, 263111.

(25) Costa, L.; Li-Destri, G.; Thomson, N. H.; Konovalov, O.; Pontoni, D. Real Space Imaging of Nanoparticle Assembly at Liquid-Liquid Interfaces with Nanoscale Resolution. Nano Letters 2016, 16, 54635468 .

(26) Sun, Y.; Xia, Y. Shape-controlled synthesis of gold and silver nanoparticles. Science 2002, 298, 2176-2179. 
(27) Dienerowitz, M.; Mazilu, M.; Dholakia, K. Optical manipulation of nanoparticles: a review. Journal of Nanophotonics 2008, 2, 021875-021875.

(28) Li, L.; Hutter, T.; Li, W.; Mahajan, S. Single nanoparticle-based heteronanojunction as a plasmon ruler for measuring dielectric thin films. The journal of physical chemistry letters 2015, 6, 22822286 .

(29) Wei, Q.; Qi, H.; Luo, W.; Tseng, D.; Ki, S. J.; Wan, Z.; Grcs, Z.; Bentolila, L. A.; Wu, T.-T.; Sun, R.; Ozcan, A. Fluorescent imaging of single nanoparticles and viruses on a smart phone. ACS nano 2013, 7, 9147.

(30) Daaboul, G.; Yurt, A.; Zhang, X.; Hwang, G.; Goldberg, B.; Unlu, M. Highthroughput detection and sizing of individual low-index nanoparticles and viruses for pathogen identification. Nano letters 2010, 10, 4727-4731.

(31) Scherr, S. M.; Daaboul, G. G.; Trueb, J.; Sevenler, D.; Fawcett, H.; Goldberg, B.; Connor, J. H.; Ünlü, M. S. Real-time capture and visualization of individual viruses in complex media. ACS nano 2016, 10, 2827.

(32) Zhang, W.; Huang, L.; Santschi, C.; Martin, O. J. Trapping and sensing 10 $\mathrm{nm}$ metal nanoparticles using plasmonic dipole antennas. Nano letters 2010, 10, 1006-1011.

(33) Liu, Y.; Huang, C. Z. Real-time dark-field scattering microscopic monitoring of the in situ growth of single $\mathrm{Ag} @ \mathrm{Hg}$ nanoalloys. ACS nano 2013, 7, 11026-11034.

(34) Rayleigh, L. XX. On the equilibrium of liquid conducting masses charged with electricity. The London, Edinburgh, and Dublin Philosophical Magazine and Journal of Science 1882, 14, 184-186.

(35) Hennequin, Y.; Allier, C. . d. P.; McLeod, E.; Mudanyali, O.; Migliozzi, D.;
Ozcan, A.; Dinten, J.-M. Optical Detection and Sizing of Single Nanoparticles Using Continuous Wetting Films. ACS Nano 2013, 7, 7601-7609.

(36) Karpitschka, S.; Dietrich, E.; Seddon, J. R. T.; Zandvliet, H. J. W.; Lohse, D.; Riegler, H. Nonintrusive Optical Visualization of Surface Nanobubbles. Phys. Rev. Lett. 2012, 109, 066102.

(37) Patterson, E. A.; Whelan, M. P. Optical signatures of small nanoparticles in a conventional microscope. Small 2008, 4, 1703-1706.

(38) Patterson, E. A.; Whelan, M. P. Tracking nanoparticles in an optical microscope using caustics. Nanotechnology 2008, 19, 105502.

(39) GINESTE, J.-M.; Macko, P.; Patterson, E.; Whelan, M. Three-dimensional automated nanoparticle tracking using Mie scattering in an optical microscope. Journal of microscopy 2011, 243, 172178.

(40) Attota, R.; Kavuri, P. P.; Kang, H.; Kasica, R.; Chen, L. Nanoparticle size determination using optical microscopes. Applied Physics Letters 2014, 105, 163105.

(41) Mudanyali, O.; McLeod, E.; Luo, W.; Greenbaum, A.; Coskun, A. F.; Hennequin, Y.; Allier, C. P.; Ozcan, A. Widefield optical detection of nanoparticles using on-chip microscopy and self-assembled nanolenses. Nature photonics 2013, 7 , nphoton-2012.

(42) Bigall, N. C.; Hartling, T.; Klose, M.; Simon, P.; Eng, L. M.; Eychmuller, A. Monodisperse platinum nanospheres with adjustable diameters from 10 to $100 \mathrm{~nm}$ : synthesis and distinct optical properties. Nano letters 2008, 8, 4588-4592.

(43) Karpitschka, S.; Weber, C. M.; Riegler, H. Spin casting of dilute solutions: Vertical 
composition profile during hydrodynamicevaporative film thinning. Chemical Engineering Science 2015, 129, 243-248.

(44) Danglad-Flores, J.; Eickelmann, S.; Riegler, H. Deposition of polymer films by spin casting: A quantitative analysis. Chemical Engineering Science 2018, 179, $257-264$.

(45) Eickelmann, S.; Riegler, H. Rupture of ultrathin solution films on planar solid substrates induced by solute crystallization. Journal of Colloid and Interface Science 2018, 528, 63 - 69 .

(46) Köhler, R.; Lazar, P.; Riegler, H. Optical imaging of thin films with molecular depth resolution. Applied physics letters 2006, 89, 241906.

(47) De Gennes, P.; Brochard-Wyart, F.; Quéré, D. Capillarity and Wetting Phenomena, 2004.

(48) Cho, K.; Hwang, I. G.; Kim, Y.; Lim, S. J.; Lim, J.; Kim, J. H.; Gim, B.; Weon, B. M. Low internal pressure in femtoliter water capillary bridges reduces evaporation rates. Scientific reports 2016, 6, 22232.

(49) Soulie, V.; Karpitschka, S.; Lequien, F.; Prene, P. M.; Zemb, T.; Moehwald, H.; Riegler, H. The evaporation behavior of sessile droplets from aqueous saline solutions. Phys. Chem. Chem. Phys. 2015, 17, 22296-22303. 